DOI: https://doi.org/10.11144/Javeriana.upsy16-4.dfdf

\title{
Delincuencia femenina y desistimiento: factores explicativos
}

\section{Female Delinquency and Withdrawal: Explanatory Factors}

Recepción: 22 Noviembre 2016 | Aprobación: 31 Marzo 2017

\author{
Luz Adriana Aristizábal Becerra ${ }^{\mathrm{a}}$ \\ Universidad Autónoma de Barcelona, España \\ ORCID: http://orcid.org/0000-0002-3620-6276 \\ Jenny Cubells Serra \\ Universidad Autónoma de Barcelona, España
}

a Autora de correspondencia. Correo electrónico: aristizabalb@gmail.com

Para citar este artículo: Aristizábal Becerra, L. A., \& Cubells Serra, J. (2017). Delincuencia femenina y desistimiento: factores explicativos. Universitas Psychologica, 16(4), 1-14. https://doi.org/10.11144/Jav eriana.upsy16-4.dfdf

\section{RESUMEN}

En este estudio de corte cualitativo, se entrevistaron 94 mujeres que estaban o habían estado en la cárcel. Se analizaron sus relatos de vida, con el fin de responder a la pregunta: ipor qué las mujeres delinquen y por qué desisten del delito? Del análisis de contenido temático se hallaron tres categorías principales: violencia de género, vínculos afectivos y cuidado. Se encontró que haber sufrido violencia de género puede ser un factor explicativo del delito, los vínculos afectivos y el cuidado del otro pueden ser factores movilizadores hacia el delito y hacia el desistimiento del mismo. Los hallazgos sugieren que las mujeres han estado encarceladas desde antes de entrar a la prisión.

Palabras clave

cárcel; cuidado; delincuencia; desistimiento; mujer; violencia de género; vínculos afectivos.

\section{ABSTRACT}

In this qualitative study, 94 women who were or had been in prison were interviewed. Their life stories were analyzed in order to answer the question: why women commit crime and why they give up crime? From the analysis of the thematic content were found three main categories: gender violence, affective bonds and care. It was found that having suffered gender violence can be an explanatory factor of the crime, the affective bonds and the care of the other can be motivating factors towards the crime and towards the abandonment of the same. The findings suggest that women have been incarcerated since before entering prison.

Keywords

prison; care; delinquency; withdrawal; woman; gender violence; affective bonds.

En las últimas décadas, se ha observado un incremento de conductas delictivas en mujeres (Azaola \& Yacamán, 1996; Contreras, 1998; González, Irarrázabal, \& Barrientos, 2016; Hernández, 2010; Mahmood, Tripodi, Vaughn, Bender, \& Schwartz, 2012; Nielsen, 2016; Ribas \& Martínez, 2003; Rodríguez, 2003; Rodríguez, Romero, Durand-Smith, Colmenares, \& Saldívar, 2006; Romero \& Aguilera, 2002; 
Salinas, 2014; Yagüe, 2007), situación que llamó la atención de las autores del presente trabajo y las llevó a suponer que obedece a un cambio social y no a una cuestión individual. Diversos estudios señalan que por lo general las mujeres que llegan a la prisión se caracterizan por ser desempleadas, con ingresos precarios, adultas jóvenes con hijos, de baja escolaridad, con trayectorias familiares delictivas (Azaola \& Yacamán, 1996) que carecen de poder y han sido violentadas la mayor parte de sus vidas (Carrillo, 2012).

Pedroza de la Llave y García (2003) manifiestan que factores como la violencia, las adicciones, la falta de oportunidades, la dependencia y la carencia extrema son elementos que hacen a las mujeres mucho más propensas a cometer delitos. Otros autores han centrado su atención en el estudio de cuestiones tales como la salud, la inteligencia, la exposición a la presión económica y un ambiente familiar desfavorable que este colectivo describe (Azaola \& Yacamán, 1996; Galván et al., 2006). También, se han examinado las formas en que las mujeres cometen delitos, la especificidad de la delincuencia femenina, las características personales de las mujeres delincuentes y los factores causales que distinguen a las mujeres delincuentes de otros grupos delicuenciales (Martínez, Carabaza, \& Hernández, 2008).

Estos hallazgos suponen una valiosa aproximación sobre las posibles situaciones que han influenciado a las mujeres para que delincan; sin embargo, se desconocen directamente sus propias narraciones. Este estudio parte de recoger los relatos de vida de las mujeres para extraer categorías explicativas que permiten generar conocimiento en este ámbito. Del análisis de contenido temático emergen tres categorías principales: violencia de género, vínculos afectivos y cuidado, que sugieren que las mujeres que delinquen han sido víctimas de violencia de género y carecen de poder, en la línea de los resultados obtenidos por Carrillo (2012). Los resultados obtenidos en este estudio apuntan a la necesidad de considerar una mirada holística, interseccional (Crenshaw, 1991; Platero, 2013; Siltanen \& Doucet, 2008) y con perspectiva de género (Almeda, 2003; Bodelón \& Aedo 2015; Yagüe, 2007; Yagüe \& Cabello, 2005), para responder a los interrogantes del porqué las mujeres delinquen y por qué desisten del delito.

Esta investigación se basa en la perspectiva socioconstruccionista, la concepción de poder de Michael Foucault (1988) y las aportaciones feministas de Bodelón \& Aedo (2015), Yagüe (2007), Almeda (2003) y Butler (2001). Los análisis feministas apuntan a la existencia de un orden androcéntrico y patriarcal que mediante las prácticas de subjetivación constituyen la identidad de los sujetos (Butler, 2001; Foucault, 1988). Cada sujeto es el correlato de sus propias prácticas de subjetivación, por lo tanto, siempre que hay nuevas prácticas que crean las condiciones de posibilidad para la transformación a través de las reglas y normas que rigen la situación, se generan nuevas formas de subjetivación que transforman a las personas. De aquí, la importancia de recuperar las narraciones de las mujeres participantes en el estudio, con el objetivo de conocer estas formas de subjetivación.

En este estudio, tambien retomamos los aportes sobre las dinámicas sociales que permean al interior de la cárcel (Calveiro, 2010; Le Breton, 1990; Ordóñez, 2006; Reidl, 1976; Rodríguez et al., 2006), sobre los efectos psicosociales de las crisis familiares (Krohn, Hall, \& Lizotte, 2009; Rivera, 2013; Schroeder, Osgood, \& Oghia, 2010) y sobre la ética del cuidado (Gilligan, 1982; Medina-Vincet, 2016).

\section{Método}

Este estudio, con un enfoque cualitativo, se enmarcó en las perspectivas socioconstruccionista (Gergen, 1985; Ibáñez \& Jimenez, 2001) y de género (Butler, 1988; Harding, 2004; Pujal, 2005). Desde esta posición, se consideró que la realidad social está construida por la acción de las personas y que por tanto hay que aproximarse a su comprensión (Burr, 1997; Gergen, 1985; Ibáñez \& Jimenez, 2001; Potter, 1998). Este acercamiento se realizó a través de un enfoque biográfico (Bertaux, 1999; Cornejo, 
Mendoza, \& Rojas, 2008) con entrevistas en profundidad sobre los relatos de vida de las mujeres entrevistadas.

En cuanto al término que se usó en este estudio: relatos de vida, en preferencia de historias de vida, se destaca el aporte del sociólogo norteamericano Norman K. Denzin (1970), quien propuso una distinción entre life story (relato de vida) y life history (historia de vida), designando al primero como la historia de una vida tal como la cuenta la persona que la ha vivido y el segundo a estudios de casos sobre una persona determinada, incluyendo no solo su propio relato de vida, sino también otras clases de documentos. Se optó por los relatos de vida por los objetivos de investigación y porque interesa resaltar la experiencia subjetiva de las mujeres que han estado en prisión, para ver cómo ellas se describen y construyen a sí mismas. Esto en concordancia con Cornejo et al. (2008) y Bertaux (1999), quienes refieren que los relatos de vida son una herramienta incomparable de acceso a lo vivido subjetivamente, y la riqueza de sus contenidos es una fuente de hipótesis inagotable, pese a las dificultades metodológicas que se puedan presentar en la recolección y en el análisis.

\section{Participantes}

En total, se entrevistaron 94 mujeres, de las cuales 81 se encontraban en la prisión en el momento de la entrevista y 13 habían estado encarceladas en algún momento de sus vidas. El tamaño de la muestra no se fijó a priori; se realizó un muestreo no probabilístico opinático o intencional, donde se buscaron perfiles que atendieran a la diversidad de situaciones personales y psicosociales respecto al delito (nacionalidad, edad y tipología delictiva), pero finalmente este estuvo sujeto a la decisión voluntaria de cada una de las mujeres que fueron convocadas. Tal como lo propone MartínezSalgado (2012, p. 615), las entrevistadas fueron nombradas "invitadas a participar", resaltando que la aceptación de cada informante fue un elemento conceptualmente imprescindible y éticamente intrínseco.

El interés fundamental no fue la medición, sino la comprensión de los fenómenos y los procesos sociales en toda su complejidad; es de primordial importancia el lugar que los participantes ocuparon dentro del contexto social, cultural e histórico del que forman parte (Crouch \& McKenzie, 2006); de ahí, el interés por conocer en profundidad los relatos de vida de diferentes mujeres en diferentes contextos. Las mujeres entrevistadas estaban, o estuvieron, en las prisiones de Barcelona (España), Montevideo (Uruguay), Oaxaca (México), Florencia, Neiva y Medellín (Colombia), y procedían de 15 naciones diferentes: España, México, Colombia, Portugal, Chile, Malí, Ecuador, Argentina, Uruguay, Paraguay, República Dominicana, Costa Rica, Perú, Guatemala y Brasil. Entre ellas, una mujer peruana con una segunda nacionalidad canadiense y una mujer colombiana con una segunda nacionalidad estadounidense (Tabla 1 ).

\section{TABLA 1}

Características de las mujeres entrevistadas

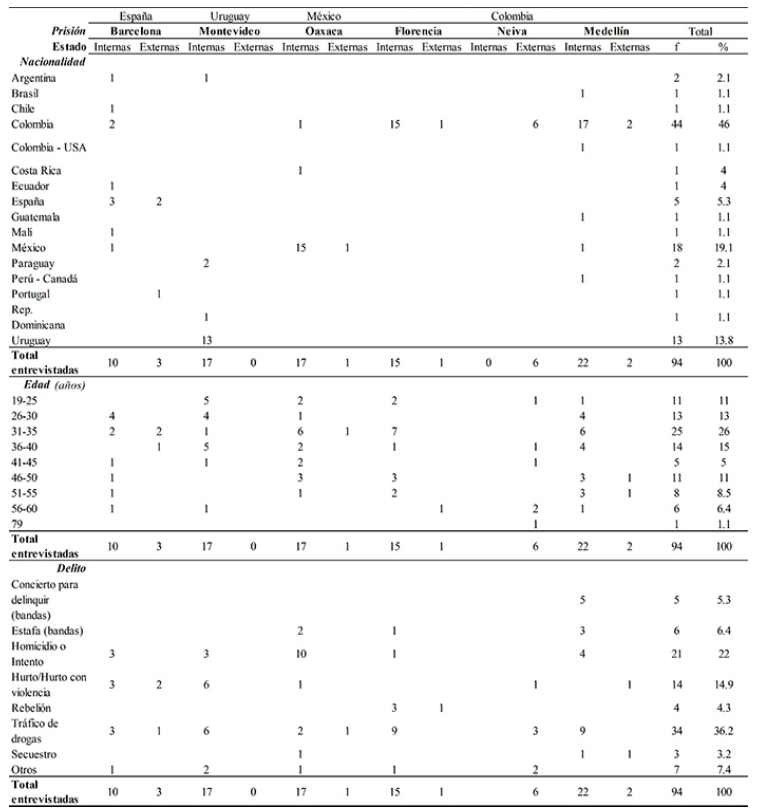

Fuente: elaboración propia. 


\section{Materiales y procedimiento}

La recolección de la información se realizó durante el tiempo comprendido entre el mes de abril del año 2015 y el mes de marzo del año 2016; sin embargo, seis meses antes se inició el proceso de contacto con universidades y/o institutos que estuvieran trabajando proyección social en las cárceles y que de alguna forma estaban relacionadas con el trabajo de las investigadoras autoras de este estudio. De esta manera, se realizó el acercamiento al centro penitenciario de Montevideo (Uruguay), a través de la Universidad de La República; a la cárcel de Tanivet en Oaxaca (México), a través de la Universidad Autónoma Benito Juárez y la Asociación Civil Mujeres unidas en torno al género, la equidad y la reivindicación (MUGER AC); a la cárcel San Cristobal de Medellín (Colombia), a través de la Universidad Nacional, Abierta y a Distancia; a la cárcel El Cunduy de Florencia (Colombia), a través de la Fundación Génesis Verde: cambio climático, postconflicto y buen gobierno y a la cárcel en Barcelona (España), a través de la Universidad Autónoma de Barcelona.

Las entrevistas se llevaron a cabo en la biblioteca de cada cárcel o en una oficina asignada por la misma dirección del Instituto Penitenciario y Carcelario. En cuanto a las mujeres que ya se encontraban fuera de la cárcel, estas fueron entrevistadas en lugares públicos o en sus respectivas viviendas, según la elección de cada entrevistada. Todas las mujeres que previamente habían sido seleccionadas por la administración de cada centro penitenciario (de acuerdo a la intención de diversificación de la muestra) recibieron la información verbal tanto en lo referido al contenido y objetivos de la investigación como respecto a las modalidades y procedimientos que implicaría la participación de cada una. Se informó que se requería grabar sus voces en los encuentros, dada la forma de análisis con que se trabajaría; también se les explicó el procedimiento realizado para llegar a contactarlas, respondiendo a sus inquietudes y aclarando que sus datos personales serían conocidos solo por los investigadores y sus testimonios serían compartidos con anonimato. Todo este proceso quedó materializado en la firma de un consentimiento informado que previamente fue revisado por la comisión académica y de ética del doctorado del Departamento de Psicología Social de la Universidad Autónoma de Barcelona, a partir de los aspectos éticos pronunciados en la Declaración de Helsinki y otros (Acevedo, 2002).

Una vez reconocido el grupo de participación, se realizó una entrevista en profundidad con cada una; la duración máxima por encuentro fue de 4 horas y la mínima de 30 minutos, con un promedio de 2 horas por cada uno. Con algunas se hizo necesario realizar dos encuentros debido a la amplitud de sus testimonios. La principal dificultad metodológica fue conseguir la entrada a las prisiones, que de por sí es una institución hermética. Sin embargo, una vez pasado el filtro institucional, se encontraron muchas mujeres interesadas en compartir sus relatos de vida. Por otro lado, se encontraron dificultades para hallar los datos de mujeres que ya estaban en libertad, en tanto que tratan de borrar las huellas de su paso por la cárcel. Se contactó a este colectivo a través de la técnica bola de nieve, a partir de contactos personales y profesionales de las investigadoras, al igual que las mismas referencias dadas por las mujeres que estaban internas.

\section{Análisis de contenido temático}

Se procedió al análisis de contenido temático como herramienta que permite sistematizar información mediante inferencias a partir de los datos obtenidos (Krippendorf, 2004). De los diferentes tipos de análisis de contenido, se optó por el análisis categorial cualitativo, en el que el contexto ocupa un lugar central. Por un lado, se tiene el contexto del texto: el material que se analiza; y, por otro, el contexto social, que hace referencia a las condiciones de posibilidad, de producción del texto (Vázquez, 1996). Siguiendo al autor, el análisis de contenido categorial consiste en que 
(...) partiendo de datos textuales, se trata de ir descomponiendo el texto en unidades para, posteriormente, proceder a su agrupamiento en categorías siguiendo el criterio de analogía. Es decir, considerando las similitudes o semejanzas que existan entre estas en función de criterios pre establecidos según los objetivos de investigación y/o análisis. (p. 49)

Para el análisis, se procedió primero a un preanálisis, organizando el material y definiendo los ejes en función de los objetivos de estudio, para proceder a la etapa de codificación en la que se transformaron los datos brutos (transcripción de los relatos de vida) a datos útiles (categorías emergentes del corpus). Se transcribieron todas las entrevistas a través de Scribe Transcription Software, siguiendo los criterios de Jefferson (2004). Se registraron los datos personales, entre ellos la nacionalidad, la edad y la tipología delictiva, en una base de datos de Excel donde se le otorgó un código a cada entrevistada para poder indentificarla rápidamente, el mismo código fue incluido en los archivos de Word digitados. Cada código de identificación señalaba las dos primeras letras del país donde fue entrevistada (ES, UR, ME, CO), seguidamente la numeración de la prisión donde está o estuvo recluida (P1: Barcelona ES, P2: Montevideo UR, P3: Oaxaca ME, P4: Florencia CO, P5: Neiva CO, P6: Medellín CO), o si estaba fuera de la prisión, se le otorgaba la misma numeración pero antecedida por una "L" y finalmente un número consecutivo dependiendo de la cantidad muestral en cada prisión. De aquí en adelante, se podrá identificar "ES(P1)02" como la segunda informante que se entrevistó en la prisión de Barcelona, España, o CO(L5)05 como la quinta informante que se entrevistó fuera de la prisión de Neiva, Colombia. Esto permitió ordenar la información y facilitar el manejo de los datos.

Se efectuó un análisis de contenido a través de ATLAS.ti Software, centrando la atención en el mensaje fruto del diálogo interpersonal producido en cada una de las entrevistas a través de la nube de palabras, lo cual proporcionó valiosas inferencias sobre las condiciones de producción y recepción (Gondim \& Bendassolli, 2014; Spini, Elcheroth, \& Figini, 2009).

\section{Resultados y discusión}

En el análisis de contenido se identificaron 21 códigos que se agruparon en tres categorías: 1. Violencia de género (VG); 2. Vínculos afectivos (VA) y 3. Cuidado (C). Durante nueve meses se realizó el proceso de transcripción y de codificación línea por línea que fue derivado de forma independiente. Algunos fragmentos de las narraciones fueron considerados como códigos relevantes, dado que eran utilizados en diferentes códigos (o categorías) a la vez (Tabla 2).

TABLA 2

Categorías y subcategorías de análisis

\begin{tabular}{lc}
\hline Categorias y subcategorías & F \\
\hline Violencia de género & 464 \\
Maltrato fisico & 123 \\
Abuso sexual & 128 \\
Otros tipos de abusos & \\
Vínculos afectivos & 2100 \\
Hijos(as) & 1787 \\
Madre & 390 \\
Pareja & 694 \\
Dios & 441 \\
Amigos(as) & \\
Cuidado & 3667 \\
Responsabilidad & 563 \\
Libertad & 388 \\
Ayudar
\end{tabular}

Fuente: elaboración propia.

Todos los códigos se analizaron por contenido temático. La frecuencia de los códigos se presentó a partir de la sumatoria total de las palabras literales (o relacionadas con el código principal) encontradas en todos los relatos. En este estudio, se dio la libertad de que cada mujer contara su relato de vida de forma abierta y de ahí la importancia del encuentro de palabras en diferentes territorios. 


\section{Violencia de género}

La violencia estuvo presente en una o varias etapas de la vida relatadas por todas las participantes. Algunas de ellas expresaron de entrada que nunca habían sido violentadas, pero en sus narraciones se hizo evidente la violencia de género. En su mayoría (92\%) describieron experiencias de maltrato físico $(\mathrm{f}=464)$.

(...) a los 20 años me fui con un señor que es el papa de mis hijas, pero no enamorada, sino por irme de la casa; yo pensaba que solo se trataba de cocinar y hacer los deberes de la casa que mi mamá hacía, pero desafortunadamente duré como 25 años con él... eso fue un maltrato físico de parte de él a cada rato, que me aguanté por mis hijas, porque estaban muy pequeñas..., pero después me fui aburriendo y aburriendo y aburriendo, hasta que me le aguanté todo, todo, hasta que ya no pude más; ya me exploté y ya no pude más. Fue una tristeza muy grande [llanto]. (CO(L6)08 ${ }^{1}$ )

Abuso sexual $(f=123)$ : "A veces vienen esos recuerdos, cuando él me metía su pene a la boca cuando yo era niña... (...) y después mis hermanos quisieron seguir haciendo lo mismo conmigo" (ES(L1)02).

Y otros abusos de poder (maltrato psicológico, inhibición de la voluntad, supresión del desarrollo de la subjetividad) ( $f=128):$ “...) la gente es mala..., yo no sé por qué a mí me pasan estas cosas..., no tengo ni idea por qué me aguanté tanto... (...), él se parecía a mi padrastro que me cogía y hacia conmigo como se le daba la gana y pues ella [madre] no me creía" (ME(P3) 16).

Este tipo de relación, limitaba su capacidad de opinar, de actuar e incluso de sentir, Toledo Jofré (2012) lo llamaría sujeto del sufrimiento social. Donde la subjetividad porta las marcas de la dominación social, el sujeto está sometido a la dependencia y al control y finalmente es víctima de la estructura social (Franssen, 1997). Tal como lo han señalado otros autores, la violencia de género impactó de manera negativa la identidad de las mujeres y su bienestar social, por el hecho de ser mujeres (Higonnet, 2007; Human Right Watch, 2008; Long, 2009).
Una minoría (8 \%) expresaron que no habían experimentado violencia de género, pero justo se observaron otras prácticas violentas como el abandono, el hacerla cómplice del delito y el control.

(...) no, mis padres nunca me pegaron, ni tampoco los vi tratarse mal entre ellos, es más, ellos nunca estaban en casa, siempre me dejaban con la nana..., (...), tampoco mi pareja, él me trataba muy bien..., mmm... solo que no me gustaba cuando me hacía viajar con él para despistar a la policía, como si fuéramos una familia que va de paseo y llevar de eso en el carro. (CO(P6)22)

En general, las mujeres se refirieron a estas prácticas violentas como parte de su cotidianidad hasta el punto de naturalizarlas. Muchas de ellas experimentaban estos hechos como ciclos que tendían a repetirse en diferentes entornos: pasar del ciclo violento familiar al escolar, luego al ciclo violento con sus parejas, al laboral, entre otros. Estos hallazgos confirman lo dicho por Aristizábal, Gurri, Molina y Sánchez (2015), que aunque se evidencian mejores oportunidades para las mujeres en el espacio público, en el privado es clara la persistencia de la desigualdad de género. También se confirma lo expresado por González et al. (2016), Bodelón \& Aedo (2015), Yagüe (2007) y Almeda (2003), al referir que la violencia de género, producto de una sociedad patriarcal, está manifiesta, en general, en la mayor parte de las mujeres que han llegado a la prisión, y en particular en todas las mujeres entrevistadas en el presente estudio.

\section{Vínculos afectivos}

De los relatos de vida de las mujeres se desprende que la comisión del delito se relaciona con los vínculos afectivos. Así, se encontraron mujeres que mataron o intentaron matar en defensa propia, ante la agresión de un hombre (por lo general denominaban a su compañero, esposo, novio como ese hombre) o en defensa de sus hijos (p. ej., el padrastro que intentó abusar sexualmente de su hija). También se identificaron mujeres que transportaban droga, porque era 
el trabajo de su pareja quien les había pedido seguirlo o como vía de auxilio o escape para encontrar dinero y salir del entorno de violencia en el que se encontraban o por encontrar los medios para suplir las necesidades de sus seres cercanos vulnerables (por lo general hijos y padres). Así se identificó en el análisis de cada uno de los delitos, donde siempre fue visible la presencia de un sujeto vincular cercano.

En primera instancia, el vínculo más fuerte y más pronunciado era el relativo a los hijos(as) (f $=2100$ ), dado por la necesidad de protección y cuidado que en sus narraciones expresaron como importantes: "Yo sé que no debía haber hecho eso..., pero fue muy duro para mí saber que él (padrastro) le estaba haciendo a mi hija lo mismo que me hizo a mí (abuso sexual)..., yo no lo iba a permitir (...), por eso lo hice (homicidio)" (ME(P3)11).

Estuvo presente el vínculo con sus madres ( $\mathrm{f}$ $=1787)$, que además de señalar su identificación como madres, también las remitía al rol desempeñado por sus mamás en su desarrollo: "Mi mamá nunca me quiso, yo creo que yo siempre fui un estorbo para ella..., todo lo que yo hacía estaba mal.., y en últimas para deshacerse de mí, me regaló. (...) Yo sé que no he sido buena madre... y a veces me duele pensar que mis hijos están corriendo la misma suerte que yo" (ME(P3)03).

En los relatos de vida también se encontró este tipo de vínculos que conducía a la acción de desistir del delito:

(...) tengo una nena de 7 años, otra de 23, otro de 20, dos nietos que me quieren, unos padres que se están haciendo mayores, que me van a necesitar, y creo que ya toca parar, parar de todo lo malo que he hecho y empezar de nuevo. Volver a nacer, por decírtelo de alguna manera. Entonces, Dios me está dando una oportunidad. (ES(P1)02)

En este caso, la mujer española que había sido inducida por su pareja a robar y aparentemente suplir las necesidades compartidas de consumo de drogas, posteriormente, fue impulsada por las relaciones con sus hijos y familiares para quienes se sentía necesaria, direccionó el rumbo de sus acciones, buscando ayuda para liberarse de la adicción y más tarde salir de la cárcel.

Se hizo evidente el código pareja, que representaba su compañero sentimental, en su mayoría maltratante. Muchas mujeres lo llamaban por su nombre propio y otras sencillamente como "ese o aquel hombre". En este punto, al igual que con sus hijos y madre, este vínculo condujo a acciones positivas y negativas:

(...) Fui muy tonta... Creí que con él había encontrado el cielo y en ese momento no pensé en nada más, no pensaba bien lo que estaba haciendo. Yo siempre he sido muy impulsiva y prácticamente he hecho lo que he querido con mi vida, pero cuando uno no piensa y se deja llevar por lo que diga el otro, entonces pasa esto, terminé haciendo todo lo que él quiso (...). Yo no soy ladrona y nunca lo he sido, no sé por qué me dejé llevar... tal vez por amor. (UR(P2)16)

En este fragmento la mujer entrevistada no se identifica como ladrona, aunque haya cometido robos, puesto que identifica esta acción como una respuesta a una relación de maltrato en la que se encuentra sujetada por amor "error que yo cometí por un hombre".

En otras expresiones (no muy frecuentes en los relatos), se halló este tipo de vinculación con la pareja encorsetando a acciones positivas:

Ahora como que me siento más aliviada, tengo una nueva pareja, tá, él está afuera esperando por mí, lo conocí cuando era niña, tá, hablo mucho con él, aunque él igual no me entiende en todo, pero al menos me escucha, tá, y me deja decir lo que quiero, y si si hay día que no quiero que venga, pues me respeta y no viene, tá. (UR(P2)11)

Del relato de la mujer uruguaya, se desprende como la relación positiva con una nueva pareja empodera al reivindicar su identidad como mujer valorada, autónoma, con la capacidad de elegir, proponer y actuar, donde de una u otra forma se sintió cuidada por otro.

Otro tipo de vínculos externos a la familia, se establecieron con Dios ( $f=694$ ) y amigos(as) ( $f$ =441). En la mayoría de los casos el vínculo con Dios se estableció o tomó fuerza al interior de las cárceles: 
(...) tenía que llegar a este lugar para hacer las pases con Dios. (ES(P1)03)

E\# iVolvería a delinquir?

MC\# No quisiera volver a robar..., no quiero volver a este lugar tan feo, pero la verdad, uno por los hijos es capaz de hacer lo que sea con tal de que a ellos no les falte nada... (...) yo pienso que lo único seguro para no volver aquí es Dios, su poder, su libertad. (CO(P6) 16)

En este tipo de relaciones de nunca acabar, muchas mujeres se definieron a sí mismas como incompletas, como un ser en falta, y es entonces cuando algunas de ellas buscaron lo infinito, lo que es perfecto, lo que no tiene límites, a quien históricamente se conoce como Dios (Lobo, 2002). De todos los vínculos manifiestos en sus relatos de vida, este vendría a ser el único vínculo que no encorseta a una acción negativa, y por lo general se estableció durante el tiempo que estuvieron en la cárcel, donde todos los recursos con los que creían que contaban se habían hecho invisibles.

Para los amigos(as) ( $\mathrm{f}=441)$, tuvieron en sus narraciones dos expresiones: por una parte, los que fueron como enemigos (influencias que las orientan al delito) y por otra los que contribuyeron en el soporte de sus crisis y a su vez las condujeron a salir de la prisión.

Si yo no la hubiera escuchado a ella, pero me puse a hacerle caso y aquí estoy.(UR(P2)03)

$\mathrm{Y}$ si yo, con este proceso no me hubiera encontrado a esa terapeuta que trabajaba allí (en la cárcel) para aquel entonces, y a la que le hicieron la vida imposible por confiar en las personas, yo hoy no estaría aquí [fuera de la cárcel]. Bueno, el caso es que... porque esa persona empezó a tratarme desde lo que yo era, no desde "cuando seas persona te trataré". iNo! "Ahora eres persona, ahora te escucho y por tanto te respeto". Y ahí empecé a cambiar. (ES(L1)01)

Del análisis se desprende la importancia de los vínculos afectivos como factor explicativo del porqué las mujeres delinquen y por qué desisten. En sus narraciones construyen los vínculos afectivos como potentes agentes movilizadores en ambos sentidos. De alguna manera, en sus relatos los vínculos que dearrollan las encorsetan a dos vías de acciones: positivas o negativas. Hasta el momento, se ha empleado el término vínculos que encorsetan, el cual surgió del análisis de los vínculos afectivos que establecieron las mujeres entrevistadas. Si nos remitimos a la definición que la Real Academia Española (RAE, 2015) encontramos que define el corsé como 'la limitación o constricción impuesta a una forma de actuar', y el término encorsetar es entendido por el Diccionario Manual de la Lengua Española (2007) como 'limitar la libertad o someter una cosa a unas normas demasiado rígidas'. Así, se usó el concepto vínculos que encorsetan para hacer referencia a toda relación afectiva establecida entre personas, con una fuerte carga emocional y que movilizan, a través de las condiciones de subjetivación que se establecen en la relación, hacia acciones positivas o negativas. Este análisis presupone la idea de que las mujeres estaban encarceladas antes de entrar a la prisión, a través de vínculos que las encorsetaron en tanto que estaban reguladas por las normas sociales y los mandatos de género y las expectativas que estos generan.

Estos hallazgos se añaden a los aportes de Delgado et al. (2006), Mora, Aristizábal y Victoria (2008) y Aristizábal et al. (2015), afirmando que los vínculos afectivos no solo favorecen la emergencia para el abuso sexual infantil, el intento de suicidio, la desigualdad de género, sino también para la inserción en conductas delictivas (que según este estudio sería en mujeres), lo cual es acorde con lo mencionado por Estrada, Rodríguez, Cerros y Solano (2015), Rivera (2013), Justicia y Cantón (2011), Schroeder et al. (2010) y Krohn et al. (2009), quienes plantearon desde diferentes enfoques cómo las relaciones con padres y/o seres cercanos ejercen una gran influencia en comportamientos problemáticos y/o actividades delictivas.

\section{Cuidado}

Por lo general, las acciones de las mujeres entrevistadas estaban dadas por la responsabilidad de cuidar del otro; en su mayoría, por cuidar de personas vulnerables 
para quienes ellas se sentían necesarias. Las mujeres, según Gilligan (1982), privilegian los vínculos con los demás, las responsabilidades en el cuidado por encima del cumplimiento abstracto de deberes y del ejercicio de derechos. En primer lugar, el verbo tener $(\mathrm{f}=3667)$ y todas sus expresiones temporales (tuve, tenía, tengo, tendré) estaba pronunciado en un contexto de obligación, responsabilidad y/o compromiso ( $\mathrm{p}$. ej., Tengo que cuidar de mis hijos, tengo que salir adelante), responsabilidad que a su vez las identifica como madres, hijas, esposas, amigas: "Yo veía a mi hija con el labio leporino y yo..., yo no podía soportarlo..., yo tenía que encontrar la forma de pagar esa cirugía (...) yo no contaba con el apoyo de nadie..., su papá no existía (...) eh, entonces accedí a hacer lo que hice" (ME(L3)01).

Esta mujer fue movida a traficar con droga por la responsabilidad sentida frente al bienestar de su hija, sin embargo, este corsé fue movido a partir de otros vínculos que la identificaban como mujer abandonada (su esposo la había dejado), mujer pobre (sin recursos económicos para sopesar las necesidades de su familia), sola (juicio por parte de sus familiares y amigos frente a sus decisiones) por cuanto la discriminación social la había llevado a ocultar su sufrimiento y sentirse sola en el esfuerzo por salir del mismo.

Seguidamente, se encuentra como subcategoría la palabra libertad ( $\mathrm{f}=563$ ). Antes de llegar a la prisión, el deseo de libertad hacía referencia a la acción de escapar frente una situación violenta o que superaba sus recursos para enfrentarlo. Así se encontraron mujeres que huyeron de sus casas parentales, escapando de una violencia y que finalmente llegaron a la casa de sus nuevas parejas para repetir la historia. Otras, que escapando de sus parejas llegaron al delito y en esa dirección a la cárcel.

¿Qué, qué creo que me llevó a cometer el delito? ... mmm... pues el necesitar escapar de la situación que estaba huyendo,... y huyéndole a una cosa me metí en algo peor... ¿Si entiende? Yo quería mi libertad, y huyéndole a ese hombre... hice lo que hice... (...). Eso fue yendo de una cosa a la otra, porque... yo me fui con el (exesposo) de mi casa no por amor, sino huyéndole a mi mamá, a los maltratos. (ES(P1)05)

En ese antes, su movilizador de libertad estaba descrito como su deseo de librarse de algo que las oprimía, y una vez llegan a la cárcel, conciben la libertad como la opción de manejar su espacio y su tiempo para cuidar del otro, específicamente del que consideran vulnerable: "Esto es muy duro...(...), lo más duro es saber que mis hijos están creciendo y yo no puedo verlos, ni cuidarlos..., ni escuchar cuando el más bebé diga mamá... (...). Yo necesito salir de aquî" ES(P1)03.

Dentro de los motivadores del desistimiento, también encontramos el verbo ayudar ( $\mathrm{f}=$ 388) como un potencializador de conductas que estuvieron direccionadas hacia el cuidado del otro, aunque muchas veces violentaron sus propios límites, intentando ingresar droga a las prisiones u otras acciones delictivas que las llevó a la cárcel. Este verbo las lleva a construirse como sujetos sociales que requieren la cooperación de unos con otros y que a su vez otorgan esperanzas en el vivir cotidiano (De la Garza, 1992; Gilligan, 1982; Murillo \& Hernández, 2016; Zemelman \& Martínez, 1987).

(...) me insistió tanto hasta que me convenció [ingresar droga a la prisión donde la pareja estaba]..., él me decía que solo me tenía a mí, que nadie más lo ayudaba..., y pues fue entrar aquí (cárcel) y ya se olvidó que yo existía. $\mathrm{CO}(\mathrm{P} 4) 07$

(...) yo por mis hijos daría la vida, ellos no se merecen tener que venir a hacer todas esas colas para venir a verme.., yo quiero salir pronto, quiero ayudarlos a salir adelante y que no se sientan solos. (ME(P3) 14)

Entre los movimientos diarios de la violencia de género, las mujeres entrevistadas intentaron responder a las expectativas generadas por la sociedad partiarcal, los vínculos identitarios las comprimen mediante el dictado de pautas de comportamiento y formas de sentir que vienen marcadas por las normas hegemónicas. Algunos vínculos las llevan a la cárcel cuando tratan de cambiar o escapar de una relación violenta (re)construyendo una relación negativa de sí mismas, y otros vínculos las movilizan a 
responsabilizarse de otros (normalmente hijos o personas dependientes) (re)construyéndose como capaces. Esto dependerá, como lo decían Berger y Luckman (1989), de sus condiciones de existencia, producto de las experiencias acumuladas a través de la historia.

\section{Consideraciones finales}

En primer lugar, el análisis de contenido temático apunta a que las mujeres que se han entrevistado en los diferentes países ya estaban presas antes de entrar a la cárcel como víctimas de la violencia de género y encorsetadas por vínculos afectivos relevantes en un sistema patriarcal; caen en la cárcel escapando de una relación de maltrato, y en esta huída se orientan al delito. Se consideró, pues, que existe una clara relación entre ser víctima y ser delincuente (como son categorizadas por el sistema) y que una vez ingresan en la cárcel únicamente se tiene en cuenta la categoría delincuente. En efecto, en algunos relatos, encontramos expresiones negativas frente al trato recibido en la cárcel, el cual estaba limitado a la condición de delincuentes y no de víctimas de la violencia.

En segunda instancia, las experiencias relatadas, las subjetividades expresadas y la literatura consultada ponen de relieve cómo una determinada construcción social de lo que significa ser mujer en nuestra sociedad patriarcal las vincula al cuidado de los demás. Las mujeres, según Gilligan (1982), dan una mayor importancia a los vínculos afectivos y a las responsabilidades en el cuidado, por encima del cumplimiento de deberes y/o del ejercicio de sus propios derechos.

De las participantes se desprende que la delincuencia femenina es producto de una sociedad patriarcal. No se han encontrado diferencias entre las mujeres entrevistadas en diferentes países y de diferentes nacionalidades en cuanto a los factores que las llevaron a delinquir y a desistir del delito en algún momento en su vida. Sin embargo, sí se observan diferencias en los países en cuanto a la legislación contra la violencia de género, la dureza de las penas, las condiciones del encarcelamiento, entre otras, que se abordarán en otras publicaciones. Un cuestionamiento de las diferencias en la construcción de los géneros orientado a la producción de nuevas subjetividades igualitarias supondría un cambio emancipador en las prácticas sociales que aportaría cambios respecto a los factores explicativos de la delincuencia. La igualdad desde la perspectiva de género implicaría vincular a la población en general al cuidado del otro, realizando una distribución más equitativa, lo cual supondría un cambio en cuanto a las prácticas delictivas.

Finalmente, qué podríamos decir frente a la pregunta: ¿cuál es la clave para que las mujeres no delincan? En primera instancia, se requiere no solo ver a las mujeres sino a la sociedad con perspectiva de género. Cuando se realiza esta comprensión, se deja de victimizar a la mujer y sencillamente se tratan de crear las condiciones de posibilidad para el desarrollo de nuevos vínculos identitarios que resulten empoderadores y que les permitan decir: $\mathrm{NO}$.

\section{Agradecimientos}

A las mujeres que voluntariamente decidieron participar en este estudio compartiendo sus historias de vida. A las Universidades y Asociaciones que apoyaron el desarrollo metodológico de este estudio. A COLCIENCIAS por financiar este proceso de investigación.

\section{Referencias}

Acevedo, I. (2002). Aspectos éticos en la investigación científica. Ciencia y Enfermería, 8(1), 15-18. http:// dx.doi.org/10.4067/ S0717-95532002000100003

Almeda, E. (2003). Mujeres encarceladas. Barcelona: Ariel.

Aristizábal, L. A., Gurri, F. D., Molina, D., \& Sánchez, G. (2015). Interaction between empowerment, economic activity and mother-child bonding. International 
Journal Advances in Social Science and Humanities, 3(1), 46-57.

Azaola, E., \& Yacamán C. (1996). Las mujeres olvidadas. México: El Colegio de México.

Berger, P., \& Luckman, T. (1989). La construcción social de la realidad. Madrid: Amorrortu.

Bertaux, D. (1999). El enfoque biográfico: su validez metodológica, sus potencialidades. Proposiciones, 29, 1-23.

Bodelón, E., \& Aedo, M. P. (2015). Las niñas en el sistema de justicia penal. Anales de la Cátedra Francisco Suárez, 49, 219-236.

Butler, J. (1988). Performative acts and gender constitution: An essay in phenomenology and feminist theory. Theatre Journal, 40(4), 519-531.

Butler, J. (2001). Mecanismos psíquicos de poder: teorías sobre la subjetivación. Madrid: Cátedra.

Burr, V. (1997). Introducció al construccionisme social. Barcelona: UOC.

Calveiro, P. (2010). El tratamiento penitenciario de los cuerpos. Cuadernos de Antropología Social, 32, 57-74.

Carrillo Hernández, E. (2012). ¿Vinculadas al narco? Mujeres presas por delitos contra la salud. Desacatos, 38, 61-72.

Contreras, L. (1998). La mujer en prisión, de su trato y tratamiento. México: Instituto Nacional de Ciencias Penales.

Cornejo, M., Mendoza, F., \& Rojas, R. (2008). La investigación con relatos de vida: pistas y opciones del diseño metodológico. Psykhe, 17(1), 29-39. https://doi.org/10.4067/ S0718-22282008000100004

Crenshaw, K. W. (1991). Mapping the margins: Intersectionality, identity politics, and violence against women of color. Stanford Law Review, 43(6), 1241-1299.

Crouch, M., \& McKenzie, H. (2006). The logic of small samples in interviewbased qualitative research. Social Science Information, 45(4), 483-499. https:// doi.org/10.1177/0539018406069584

Delgado, M. C., Oviedo, M., Cabrera, E., González, A. C., Montero M. I., Charry, C. L., ... Murci, C. C. (2006). Nichos fragmentados: emergencia del abuso sexual infantil. Bogotá: Editorial Gente Nueva.

Denzin, K. (1970). The research act. Chicago: Aldine.

De la Garza, E. (1992). Los sujetos sociales en el debate teórico. En E. De la Garza (Coord.), Crisis y sujetos sociales en México (Vol. 1, pp. 15-52). México: Porrúa-CIIH-UNAM.

Diccionario Manual de la Lengua Española. (2007). Encorsetar [Catálogo Vox, 17. a ed.]. Madrid: Larousse.

Estrada, C., Rodríguez, F. J., Cerros, E., \& Solano, C. (2015). Implicaciones parentales en las conductas delictivas de adolescentes: tendencias y narrativas. Papeles de Población, $21(84), 107-132$.

Franssen, A. (1997). Balises et écueils d'une sociologie du sujet. En G. Bajoit \& E. Belin (Eds.), Constributions à une sociologie du sujet (pp. 17-50). París: LHarmattan.

Foucault, M. (1988). Tecnologías del yo. Bellaterra: ICE-UAB.

Galván, J., Romero, M., Rodríguez, E., Durand, A., Colmenares, E., \& Saldivar, G. (2006). La importancia del apoyo social para el bienestar físico y mental de las mujeres reclusas. Revista Salud Mental, 29(3), 68-73.

Gilligan, C. (1982). In a different voice: Psychological theory and women's development. Cambridge: Harvard University.

Gergen, K. (1985). The social constructionist movement in modern psychology. American Psychologist, 40(3), 266-275. https:// doi.org/10.1037/0003-066X.40.3.266

Gondim, S. M. G., \& Bendassolli, P. F. (2014). The use of the qualitative content analysis in psychology: A critical review. Psicologia em Estudo, 19(2), 191-199. http:// dx.doi.org/10.1590/1413-737220530002

González, T., Irarrázabal, T., \& Barrientos, F. (2016). Experiencias de violencia de género en una muestra de mujeres condenadas a prisión. Revista de Estudios Criminológicos y Penitenciarios, 20, 45-84.

Harding, S. (Ed.). (2004). The feminist standpoint theory reader. Intellectual and political controversies. Nueva York: Routledge. 
Hernández, R. A. (2010). Bajo la sombra del Guamúchil. México: CIESAS/IWGIA/OreMedia.

Higonnet, E. (2007). "My Heart is Cut". Sexual violence by rebels and pro-government forces in Côte D'Ivoire (Informe 19(11)A). Nueva York: Human Rights Watch. Recuperado de https://www.hrw.org/report/2007/08/02/ my-heart-cut/sexual-violence-rebels-and-p ro-government-forces-cote-divoire

Human Rights Watch. (2008). These everyday humiliations: Violence against lesbians, bisexual women, and transgender men in Kyrgyzstan (Informe). Nueva York: Autor. Recuperado de https://www.hrw.org/report s/2008/kyrgyzstan1008/

Ibáñez, T., \& Jiménez, B. (2001). Psicología social construccionista. Guadalajara: Universidad de Guadalajara.

Jefferson, G. (2004). Glossary of transcript symbols with an introduction. En G. H. Lerner (Ed.), Conversation analysis: Studies from the first generation (pp. 13-23). Philadelphia: John Benjamins.

Justicia, M. J., \& Cantón, J. (2011). Conflictos entre padres y conducta agresiva y delictiva en los hijos. Psicothema, 23(1), 20-25.

Krippendorf, K. (2004). Content analysis. An introduction to its methodology (2.a ed.). Thousand Oaks, CA: Sage.

Krohn, M., Hall, F. P., \& Lizotte, A. (2009). Family transitions and later delinquency and drug use. Journal of Youth and Adolescence, 38(3), 466-480. https:// doi.org/ 10.1007/s10964-008-9366-8

Le Breton, D. (1990). Anthropologie du corps et modernité. París: Presses Universitaires de France.

Lobo, G. (2002). Dios Uno y Trino: manual de iniciación (5.a. ed.). Madrid: Rialp.

Long, S. (2009). They want us exterminated: Murder, torture, sexual orientation and gender in Iraq (Informe). Nueva York: Human Rights Watch. Recuperado de https:/www.hrw.org/report/2009/08/17/ they-want-us-exterminated/murder-tortur e-sexual-orientation-and-gender-iraq
Mahmood, S. T., Tripodi, S. J., Vaughn, M. G., Bender, K., \& Schwartz, R. (2012). Effects of personality disorder and impulsivity on emotional adaptations in prison among women offenders. Psychiatric Quarterly, 83, 467-480. https:// doi.org/10.1007/s11126-012-9215-5

Martínez, P., Carabaza, R., \& Hernández, A. (2008). Factores de riesgo predisponentes a la delincuencia en una población penal femenina. Revista Enseñanza e Investigación en Psicología, 13(2), 301-318.

Martínez-Salgado, C. (2012). El muestreo en investigación cualitativa. Principios básicos y algunas controversias. Saúde Colectiva, 17(3), 613-619.

Medina-Vincet, M. (2016). La ética del cuidado y Carol Gilligan: una crítica a la teoría del desarrollo moral de Kohlberg para la definición de un nivel moral postconvencional contextualista. Daimon. Revista Internacional de Filosofía, 67, 83-98. https://doi.org/10.6018/daimon/199701

Mora, A., Aristizábal, L. A., \& Victoria, J. (2008). Factores de riesgo asociados al intento de suicidio en la ciudad de Neiva durante el año 2006. Modernidad, Tecnología y Síntomas Contemporáneos. Serie Conexiones Argentina, 1, 278-280.

Murillo, F. J., \& Hernandez-Castilla, R. (2016). Hacia un concepto de justicia social. Revista Iberoamericana sobre Calidad, Eficacia y Cambio en Educación, 9(4), 7-23.

Nielsen, I. L. (1 de octubre de 2016). Discovering disenfranchased and invisible women in prison. [Revisión del libro Women doing life: Gender, punishment, and the struggle for identity, por L. B. Lempert]. Sex Roles, 75, 630-631. https://doi.org/10.1007/ s11199-016-0687-x

Ordóñez, L. (2006). Mujeres encarceladas: proceso de encarcelamiento en la penitenciaria femenina de Brasilia. Revista Universitas Humanística, 61 (61), 25-32.

Pedroza de la Llave, S., \& García, O. (Comps.). (2003). Compilación de instrumentos internacionales de derechos humanos firmados y ratificados por México 
1921-2003 (Vol. 2). México: Comisión Nacional de los Derechos Humanos.

Platero, R. (2013). Marañas con distintos acentos: género y sexualidad en la perspectiva interseccional. Encrucijadas. Revista Crítica de Ciencias Sociales, 5, 44-52.

Potter, J. (1998). La representación de la realidad: discurso, retórica y construcción social. Barcelona: Paidós.

Pujal, M. (2005). El feminisme. Barcelona: UOC.

Real Academia Española. (2015). Corsé. En Diccionario de la lengua española (23a. ed.). Recuperado de http://dle.rae.es/?id=BOwe QIY

Reidl, L. (1976). Prisionalización en una cárcel de mujeres. México: Instituto Nacional de Ciencias Penales.

Ribas, M., \& Martínez, A. (2003). Mujeres extranjeras en las cárceles españolas. Revista Sociedad y Economía, 5, 65-80.

Rivera, J. G. (2013). Juventudes en América Latina: una reflexión desde la experiencia de la exclusion y la cultura. Papeles de Población, 19(75), 9-34.

Rodríguez, M. N. (abril, 2003). Mujer y cárcel en América Latina. Informe del seminario-taller Violencia contra las mujeres privadas de libertad en América Latina, México. Recuperado de http://catedraunescodh.unam.mx/cated $\mathrm{ra} /$ SeminarioCETis/Documentos/Doc basi cos/5_biblioteca_virtual/7_violencia/ $\overline{19}$.pd $\mathrm{f}$

Rodríguez, E., Romero, M., Durand-Smith, A., Colmenares, E., \& Saldívar, G. (2006). Experiencias de violencia física ejercida por la pareja en las mujeres en reclusión. Revista Salud Mental, 29(2), 59-67.

Romero, M., \& Aguilera, R. M. (2002). ¿Por qué delinquen las mujeres? Perspectivas teóricas tradicionales. Parte 1. Salud Mental, 25(5), 10-22.

Salinas, C. (2014). Las cárceles de mujeres en México: espacios de opresión patriarcal. Iberóforum. Revista de Ciencias Sociales de la Universidad Iberoamericana, 9(17), 1-27.

Schroeder, R., Osgood, A., \& Oghia, M. (2010). Family transitions and juvenile delinquency. Sociological Inquiry, 80(4), 579-604. https://oi.org/ 10.1111/ j.1475-682X.2010.00351.x

Siltanen, J., \& Doucet, A. (2008). Gender relations in Canada: Intersectionality and beyond. Toronto: Oxford University Press.

Spini, D., Elcheroth, G., \& Figini, D. (2009). In there space for time in social psychology publications? A content analysis across five journals. Journal of Community $\mathcal{E}$ Applied Social Psychology, 19(3), 165-181. https:// doi.org/ 10.1002/casp.991

Toledo Jofré, M. I. (2012). Sobre la construcción identitaria. Atenea, 506, 43-56. https://doi.org/10.4067/ S0718-04622012000200004

Vázquez, F. (1996). El análisis de contenido temático. Objetivos y medios en la investigación psicosocial (Documento de trabajo, pp. 48-61). Barcelona: Universitat Autónoma de Barcelona.

Yagüe, C. (2007). Mujeres en prisión. Intervención basada en sus características, necesidades y demandas. Revista Española de Investigación Criminológica, 4(5), 1-24.

Yagüe, C., \& Cabello, M. I. (2005). Mujeres y jóvenes en prisión. Revista de Estudios de Juventud, 5(69), 30-48.

Zemelman, H., \& Martínez, A. Q. (1987). Conocimiento y sujetos sociales: contribución al estudio del presente (Vol. 3). México: Colegio de México, Centro de Estudios Sociologicos.

\section{Notas}

1 Este es el código de identificación dado a cada mujer entrevistada, lo cual indica: las dos primeras letras en mayúscula identifican el país en el que se realizó la entrevista (ES: España, UR: Uruguay, ME: México, CO: Colombia). Seguidamente la letra P indica que fue entrevistada en la prisión y la letra $L$ indica que fue entrevistada fuera de la prisión. El número que sucede a esta letra corresponde a la ciudad específica donde fue entrevistada (1: Barcelona ES, 2: Montevideo UR, 3: Oaxaca ME, 4: Florencia CO, 5: Neiva CO, 6: Medellín 
CO. Finalmente se añade un número único consecutivo de acuerdo al número muestral en cada ciudad.

* Artículo de investigación. 